\title{
Performance Characteristics of ELISA to Detect Bovine Viral Diarrhea Virus (BVDV) Antibodies Using Colostrum
}

\author{
Caitlin J. Jenvey ${ }^{1}$, Andrew M. Weir ${ }^{2}$, Michael P. Reichel1, Peter D. Cockcroft ${ }^{1}$ \\ ${ }^{1}$ School of Animal and Veterinary Sciences, University of Adelaide, Roseworthy Campus, Roseworthy, Australia \\ ${ }^{2}$ Eltham District Veterinary Service, Eltham, New Zealand \\ Email: caitlin.jenvey@adelaide.edu.au
}

Received 29 January 2015; accepted 12 February 2015; published 13 February 2015

Copyright (C) 2015 by authors and Scientific Research Publishing Inc.

This work is licensed under the Creative Commons Attribution International License (CC BY). http://creativecommons.org/licenses/by/4.0/

(c) (i) Open Access

\begin{abstract}
Colostrum contains substantially higher concentrations of immunoglobulins (Igs) when compared with serum or milk, which may improve the diagnostic sensitivity of an antibody ELISA when using colostrum. In this study, BVD was used as a model to identify the performance characteristics of colostrum and to assess the potential for increased ELISA sensitivity when compared with serum. Blood and colostrum samples were collected from cows within two dairy cattle herds: a previously infected and BVD-vaccinated Holstein-Friesian (positive herd) herd, and a bulk-tank milk antibody negative (negative herd) Jersey herd. All samples were tested using a commercial BVDV antibody ELISA. Median sample-to-positive (S/P) colostrum ratios were significantly higher than their respective serum counterparts, and positive herd $S / P$ ratios were significantly higher than the respective negative herd values $(P<0.001)$. Using the manufacturer's recommended serum dilution (1:5) and colostrum dilution (undiluted), and a cut-off threshold $S / P$ ratio of 0.2 , diagnostic sensitivity (DSe) and diagnostic specificity (DSp) for colostrum were $100 \%$ and $70 \%$, respectively. These values increased to $100 \%$ DSe and $100 \%$ DSp with an increase in cut-off threshold S/P to 0.5. At a sample dilution of 1:100, the DSe of colostrum was $90 \%$ and significantly higher compared with serum (DSe 17\%). Colostrum has the potential to improve identification of previously infected animals, either individually, or when using pooled samples.
\end{abstract}

\section{Keywords}

BVDV, Colostrum, ELISA, Sensitivity, Specificity

\section{Introduction}

The production of colostrum is a selective process. Maternal immunoglobulins (Igs) are transferred to the

How to cite this paper: Jenvey, C.J., Weir, A.M., Reichel, M.P. and Cockcroft, P.D. (2015) Performance Characteristics of ELISA to Detect Bovine Viral Diarrhea Virus (BVDV) Antibodies Using Colostrum. Open Journal of Veterinary Medicine, 5, 35-41. http://dx.doi.org/10.4236/ojvm.2015.52006 
mammary gland, from the dam's bloodstream, prior to parturition and in the early post-partum period. The concentration of these Igs in cattle colostrum has been found to be five [1] to ten times [2] higher than serum. Colostrum, therefore, has the potential to raise the analytical (as well as diagnostic) sensitivity of serological assays for the detection of antibodies against specific diseases.

Bovine viral diarrhoea virus (BVDV) is a pestivirus in the family Flaviviridae and is considered one of the most economically important infectious diseases of cattle worldwide [3] [4]. Infection with the virus may cause abortion and other clinical signs including fever and diarrhoea. Clinical severity is dependent upon the viral subtype, but acute infections are often accompanied by mild clinical signs. The virus is economically detrimental to both the beef and dairy industries due to an increase of $18 \%-40 \%$ [5] in abortion rates and a $22 \%$ [6] decrease in conception rates in infected cattle. Acutely infected animals mount an immune response and remain antibody positive for an extended period. Antibody (Ab)-enzyme-linked immunosorbent assay (ELISA) is the test of choice for routine diagnosis of exposure to BVDV, as it is rapid, relatively inexpensive to perform and more suitable for large scale testing [7]. The ELISA has previously been used, not only for the testing of serum [8]-[10], but also milk [7] [11]-[13].

Although the testing of individual and bulk tank milk samples has been established, there is currently little research into whether colostrum will be superior to both serum and milk. A study by Jenvey et al. [14] demonstrated a significant increase in diagnostic sensitivity when comparing colostrum with serum in Johne's disease vaccinated sheep. It is possible that using colostrum can also improve the sensitivity of a commercial BVDV $\mathrm{Ab}$-ELISA and therefore increase confidence in the absence of disease, or improve identification of exposure at the herd level when using pooled samples. The collection and testing of serum and colostrum samples from a BVD vaccinated dairy herd and a BVD bulk-milk negative dairy herd were a "proof-of-concept" study to determine the performance characteristics of a commercially available ELISA for BVDV antibodies when applied to colostrum, as compared with serum.

\section{Materials and Methods}

\subsection{Animal Selection}

Positive herd. A Holstein-Friesian dairy herd was selected as the positive herd based upon a history of previous BVDV infection, as well as the vaccination of the herd with the BVD vaccine Pestigard® (Zoetis). The herd was vaccinated annually since 2009 , and most recent vaccination was done in April 2012. Sample collection was performed in March to May 2012. The first 30 dairy cows to calve were sampled (excluding first calving heifers).

Negative herd. A Jersey dairy herd was selected as the negative herd based upon consistent low bulk tank milk antibody levels (i.e. herd was sampled 3 times per year for 6 years prior to sample collection and consistently recorded $\mathrm{S} / \mathrm{P}$ ratio results $<0.25$ ). In addition, a bulk tank milk antibody $\mathrm{S} / \mathrm{P}$ ratio of 0.0 was recorded several months after sample collection had concluded, indicating that the herd was not exposed to BVDV at the time of sampling. Sample collection was performed in August to October 2013. The first 20 dairy cows to calve were sampled (excluding first calving heifers).

Owner informed consent and project approval was obtained from the University of Adelaide Animal Ethics Committee.

\subsection{Sample Collection and Testing}

Positive herd. The colostrum samples were collected within 12 hours of calving and the blood samples were collected from the coccygeal vein 30 - 60 days post calving. Blood samples were centrifuged for 10 minutes at $1157 \mathrm{~g}$ to separate the serum. All samples were stored at $-80^{\circ} \mathrm{C}$ until testing was performed. The samples were then tested using a commercial BVDV Total Antibody ELISA (IDEXX). Individual samples were diluted (1:2, $1: 5,1: 10,1: 20,1: 40,1: 80$ and 1:100) using the diluent supplied with the ELISA kit. All samples were tested in triplicate and results expressed as sample-to-positive $(\mathrm{S} / \mathrm{P})$ ratio.

Negative herd. Colostrum samples were collected within 24 hours of calving and prior to the first milking and the blood samples were collected from the coccygeal vein between 30 - 60 days post calving. The samples were tested by Gribbles Veterinary (Palmerston North, NZ) using a commercial BVDV Total Antibody ELISA (IDEXX). Individual samples were tested as per the manufacturer's instructions for milk (undiluted) and serum (1:5 diluted), and expressed as sample-to-positive $(\mathrm{S} / \mathrm{P})$ ratio. 


\subsection{Statistical Analysis}

Frequency histograms and Q-Q plots determined that the data was non-normally distributed. A Wilcoxon signed rank test was used to identify any significant differences ( $\mathrm{R}$ version 3.0.2). A receiver operating characteristic (ROC) curve analysis was performed to determine DSe in relation to sample dilution for both serum and colostrum using the data from the positive herd. A two graph-receiver operating characteristic (TG-ROC) analysis was performed for both colostrum and serum, for a range of S/P cut-off thresholds. Diagnostic sensitivity (DSe, from the positive herd) and diagnostic specificity (DSp; from the negative herd) were defined and calculated as follows:

$$
\begin{gathered}
\mathrm{DSe}=\frac{\text { Number infected } / \text { vaccinated cows testing serum positive }}{\text { Total number infected } / \text { vaccinated cows }} \times 100 \\
\mathrm{DSp}=\frac{\text { Number of bulk tank milk negative cows testing serum negative }}{\text { Total number bulk tank milk negative cows }} \times 100
\end{gathered}
$$

\section{Results}

The median S/P ratio for colostrum collected from the positive herd was significantly higher when compared to the corresponding positive serum samples, at all dilutions $(\mathrm{P}<0.001)$ (Table 1$)$. The median $\mathrm{S} / \mathrm{P}$ ratio for colostrum collected from the negative herd was significantly higher compared to negative serum $(\mathrm{P}<0.001)(\mathrm{Table}$ 1). Comparison of samples at a 1:5 dilution showed the median $\mathrm{S} / \mathrm{P}$ ratio for the positive herd colostrum was significantly higher compared to negative herd colostrum and positive herd serum $(\mathrm{P}<0.001)$; negative herd colostrum was significantly higher compared to negative herd serum $(\mathrm{P}<0.001)$; positive herd colostrum was significantly higher compared to negative herd colostrum $(\mathrm{P}<0.001)$; positive herd colostrum was significantly higher compared to negative herd serum $(\mathrm{P}<0.001)$; positive herd serum was significantly higher compared to negative herd colostrum $(\mathrm{P}<0.001)$; and positive herd serum was significantly higher compared to negative herd serum $(\mathrm{P}<0.001)$ (Table 1$)$.

The DSe of both serum and colostrum decreased with an increase in sample dilution. Serum DSe decreased more rapidly with an increase in dilution, reaching a DSe of just 17\% at a sample dilution of 1:100 (Figure 1). The DSe of colostrum also decreased with an increase in sample dilution, but did not fall below 90\% (at 1:100 dilution) (Figure 1). Differences in DSe between serum and colostrum were statistically significant for dilutions 1:20, 1:40, 1:80 and 1:100 $(\mathrm{P}<0.05$; Figure 1$)$.

Two-graph receiver operating characteristic (TG-ROC) analysis for the serum and colostrum samples is presented in Figure 2. The TG-ROC analysis indicates the specificity (using the negative herd data) and sensitivity (using the positive herd data) at various cut off $\mathrm{S} / \mathrm{P}$ thresholds. Using the recommended serum dilution and cut-off S/P ratio of 0.2 , both DSp and DSe for serum was 100\% (Figure 2). Using undiluted colostrum and the same cut-off threshold, DSp and DSe was 70\% and 100\%, respectively (Figure 2). An increase in cut-off S/P to 0.5 increased colostrum DSp to $100 \%$, while DSe for colostrum remained at 100\% (Figure 2). From a cut-off $\mathrm{S} / \mathrm{P}$ ratio of 0.5 and upwards, colostrum showed an increasingly and significantly higher DSe compared to serum $(\mathrm{P}<0.05)$, whilst maintaining high DSp comparable to that of serum (Figure 2).

\section{Discussion}

Diagnostic sensitivity is defined as the percentage of subjects with a disease that are identified by the assay as positive for the disease [15]. This study does not attempt to identify true positive and true negative animals, but rather, it attempts to determine whether colostrum can improve the diagnostic sensitivity of an ELISA relative to serum samples. In this study, the use of colostrum as a sample demonstrated a significantly higher signal (S/P ratios) and DSe compared to serum, in particular with increasing sample dilution. Using a sample dilution of 1:100; the DSe of the ELISA when using colostrum samples compared to serum samples was $90 \%$ and $17 \%$, respectively. The DSe of the ELISA when using colostrum did not fall below $90 \%$ for all the dilutions investigated. Although the cattle in the current study were vaccinated against BVDV, this vaccination occurred following a previous outbreak of BVDV in the milking herd; therefore, the ELISA S/P results in this study are higher than what would be found in herds that had only been vaccinated (Lanyon, 2014, personal communication). When using pooled samples, colostrum would be able to detect a lower prevalence of BVDV exposure when compared to milk or serum. 


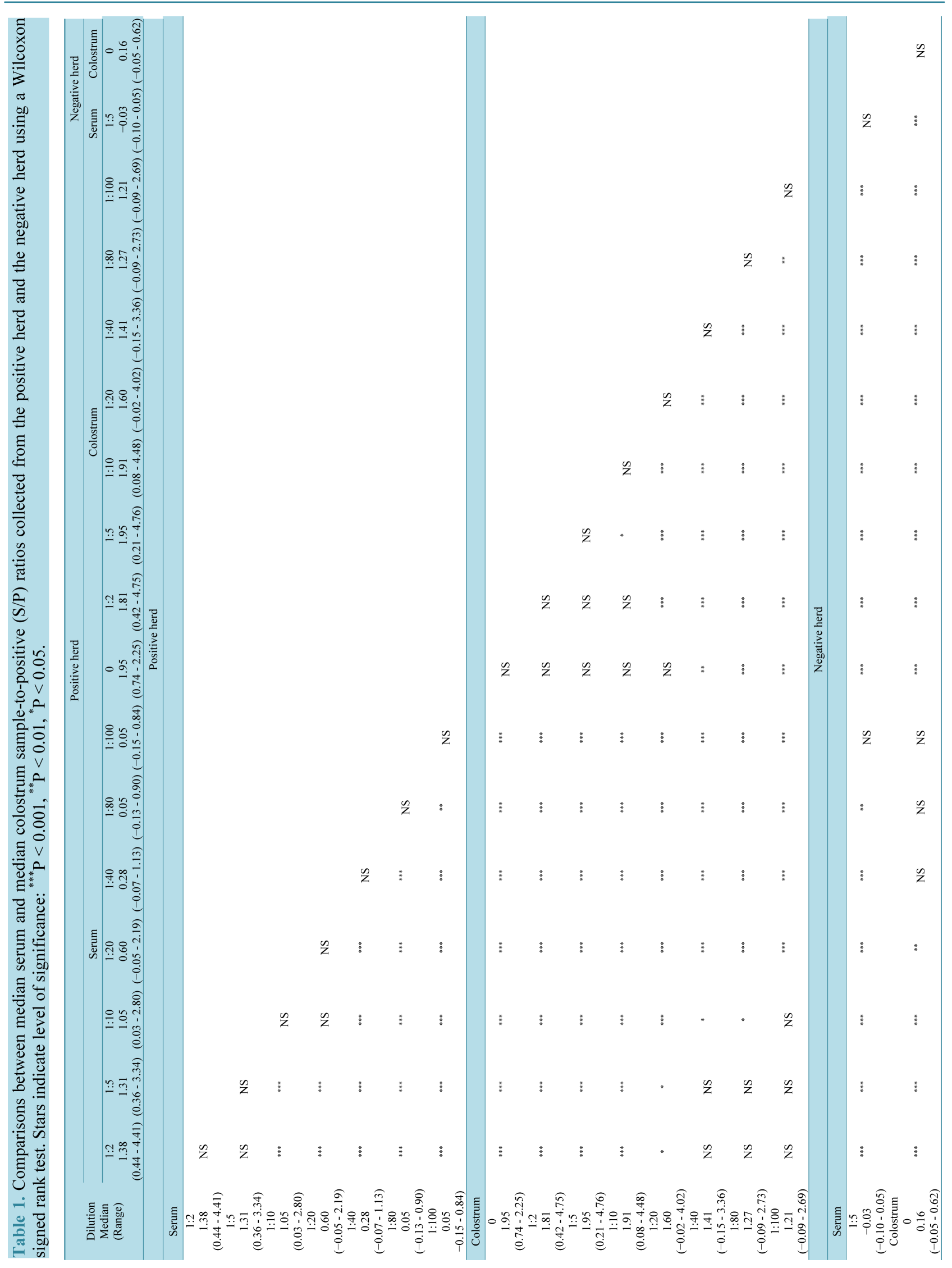




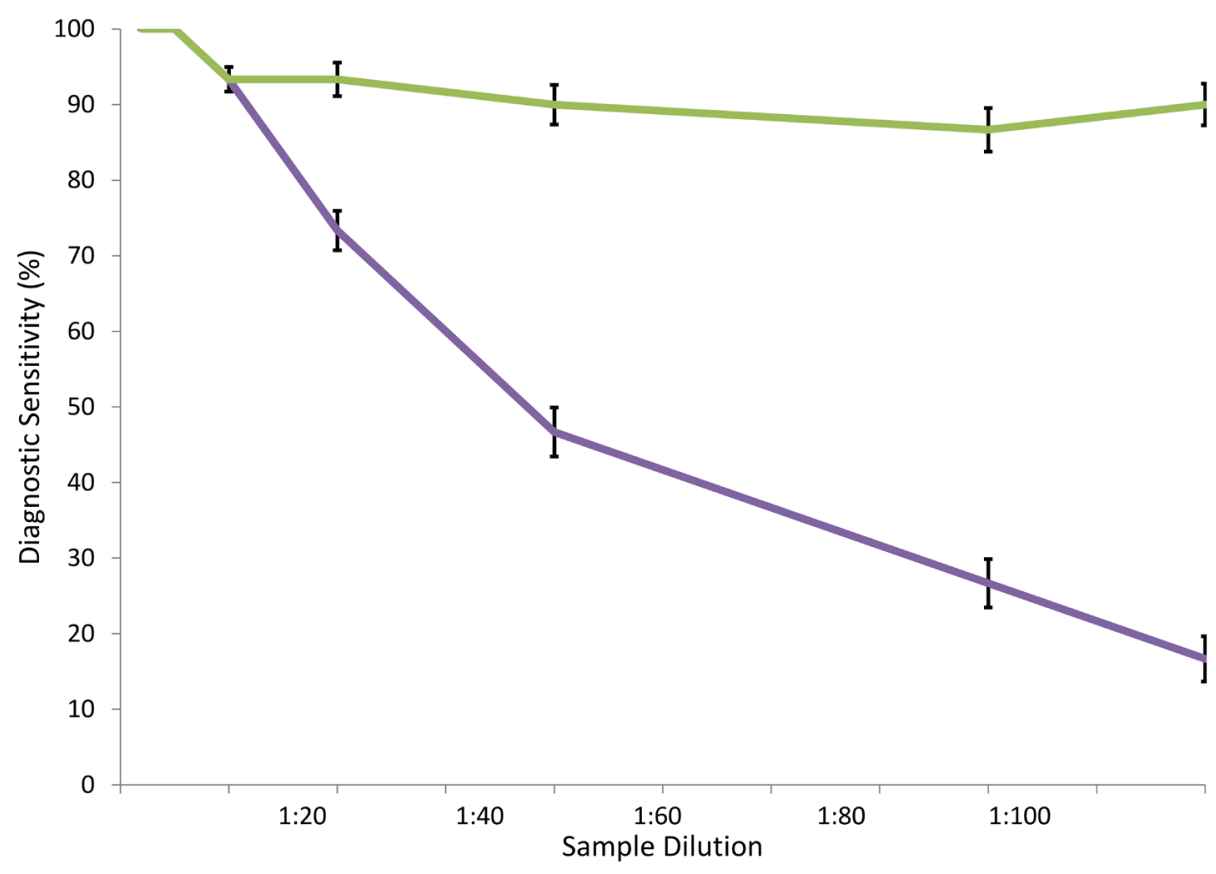

Figure 1. Diagnostic sensitivity (DSe) $\pm 95 \%$ confidence intervals, expressed as a percentage, for serum (purple) and colostrum (green) collected from the positive herd (BVD vaccinated), diluted $1: 2,1: 5,1: 10,1: 20,1: 40,1: 80$ and $1: 100$.

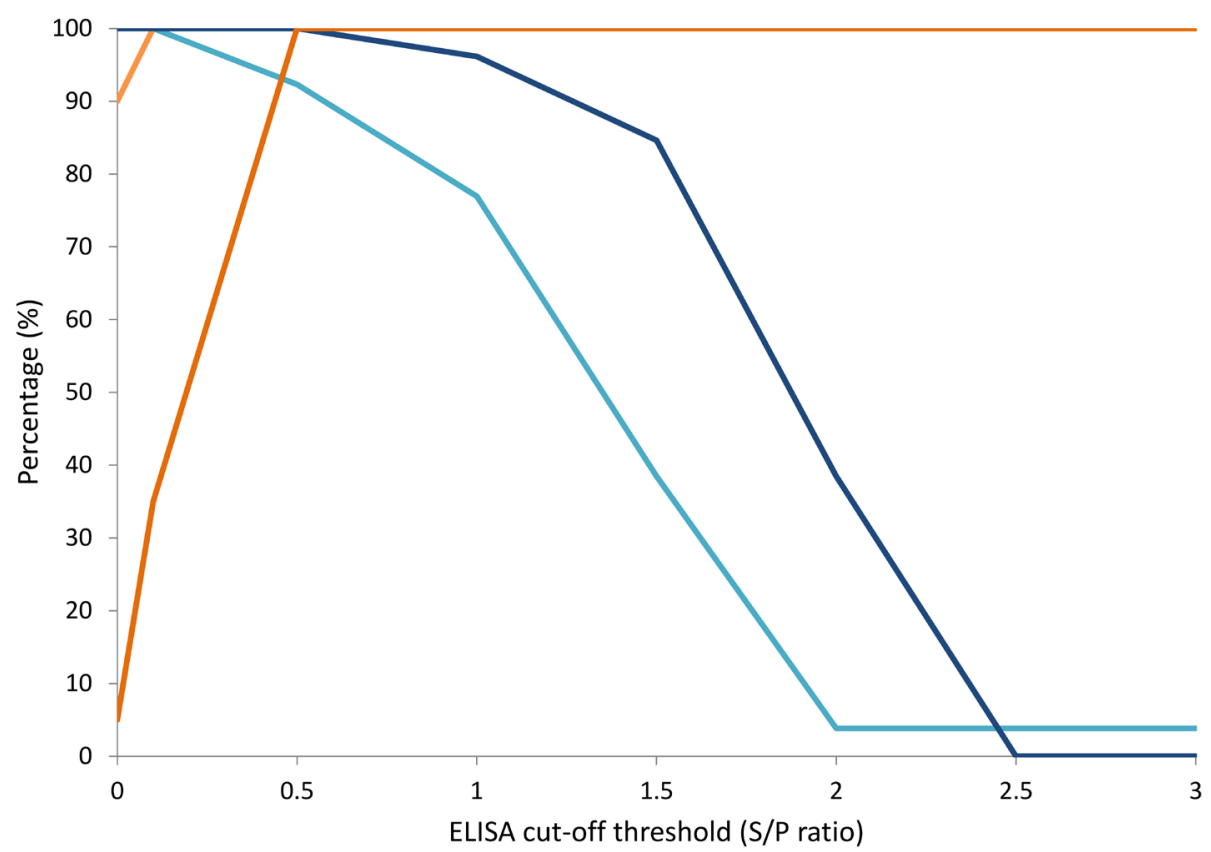

Figure 2. Two-graph receiver operating characteristic analysis of serum and colostrum using a commercial ELISA for the detection of antibodies specific to BVDV. Diagnostic sensitivity (serum: light blue; colostrum: dark blue) was calculated using sample-to-positive (S/P) ratio results from the positive herd (BVD vaccinated) and diagnostic specificity (serum: light orange; colostrums: dark orange) was calculated using sample-to-positive $(\mathrm{S} / \mathrm{P})$ ratio results from the negative herd (bulk tank ilk negative).

Using a positive cut-off of $\geq 0.5$, the DSe and DSp of ELISA testing colostrum was $100 \%$. Using the same commercial ELISA, a study by Weir et al. [13] recommended a decrease in the positive ELISA cut-off value 
from $\geq 0.3$ to $\geq 0.13$ when testing for BVDV using milk. When using the new milk cut-off value, the study found a relative sensitivity and specificity of $100 \%$ and $94 \%$, respectively [13]. A similar study by Lanyon et al. [16] found a relative sensitivity and specificity of $96.6 \%$ and $89.2 \%$, respectively, which was lower than what was observed in the study by Weir et al. [13] but most likely due to the use of the unadjusted positive cut-off value of $\geq 0.3$. Both the studies by Weir et al. [13] and Lanyon et al. [16] concluded that milk was an appropriate alternative to the testing of serum using ELISA, as the agreement between the results for both sample types was high. In our study, however, colostrum has an even better performance than reported for milk or serum with regards to the diagnostic sensitivity at higher dilutions due, most likely, to the higher concentrations of Igs in colostrum. Although, in our study, we achieved similar performance characteristics for both serum and colostrum after the ELISA cut-off threshold had been adjusted, the DSe for colostrum continued to be significantly higher compared to serum when the samples were diluted. This would suggest that in BVD monitoring programs, particularly where eradication is under consideration, colostrum samples could provide an improved diagnostic utility compared to serum or milk samples when using the antibody ELISA on larger pools of samples.

Recently, the testing of colostrum using ELISA was investigated by Zervens et al. [17] to assess the occurrence of non-specific reactions when testing colostrum for Mycobacterium avium subspecies paratuberculosis (MAP)-specific antibodies. Zervens et al. [17] found only 4\% non-specific reactions when testing colostrum collected at 0 DIM (days-in-milk). The study concluded that since few non-specific reactions occur in the ELISA using colostrum samples, colostrum would be a useful diagnostic sample for the detection of MAP-specific IgG.

\section{Conclusion}

In conclusion, the increase in diagnostic sensitivity that may be able to be achieved when testing colostrum provides an opportunity to improve the identification of infected animals, either individually, or by using pooled samples. This observation of improved test performance using colostrum compared with serum or milk with regards to diagnostic sensitivity may also find application in the diagnosis of other important (dairy) cattle diseases, such as Mycobacterium bovis (Bovine Tuberculosis), Neospora caninum, Leptospirosis and Johne's disease.

\section{Acknowledgements}

The author's would like to thank Dr. Rosemary Santangelo of IDEXX Laboratories for the supply of ELISA kits used in this study.

\section{References}

[1] Beer, A., Billingham, R. and Head, J. (1974) The Immunologic Significance of the Mammary Gland. Journal of Investigative Dermatology, 63, 65-74. http://dx.doi.org/10.1111/1523-1747.ep12678092

[2] Baumrucker, C.R., Burkett, A.M., Magliaro-Macrina, A.L. and Dechow, C.D. (2010) Colostrogenesis: Mass Transfer of Immunoglobulin G1 into Colostrum. Journal of Dairy Science, 93, 3031-3038. http://dx.doi.org/10.3168/jds.2009-2963

[3] Houe, H. (2003) Economic Impact of BVDV Infection in Dairies. Biologicals, 31, 137-143. http://dx.doi.org/10.1016/S1045-1056(03)00030-7

[4] Gunn, G., Stott, A. and Humphry, R. (2004) Modelling and Costing BVD Outbreaks in Beef Herds. Veterinary Journal, 235, 1171-1179.

[5] McGowan, M. and Kirkland, P. (1995) Early Reproductive Loss Due to Bovine Pestivirus Infection. British Veterinary Journal, 151, 263-270. http://dx.doi.org/10.1016/S0007-1935(95)80176-6

[6] Brownlie, J., Hooper, L., Thompson, I. and Collins, M. (1998) Maternal Recognition of Foetal Infection with Bovine Virus Diarrhoea Virus (BVDV)—The Bovine Pestivirus. Clinical Diagnostic Virology, 10, 141-150. http://dx.doi.org/10.1016/S0928-0197(98)00030-0

[7] Beaudeau, F., Belloc, C. and Seegers, H. (2001) Evaluation of a Blocking ELISA for the Detection of Bovine Viral Diarrhoea Virus (BVDV) Antibodies in Serum and Milk. Veterinary Microbiology, 80, 329-337. http://dx.doi.org/10.1016/S0378-1135(01)00322-4

[8] Durham, P. and Hassard, L. (1990) An Enzyme-Linked Immunosorbent Assay (ELISA) for Antibodies to Bovine Viral Diarrhoea Virus. Veterinary Microbiology, 22, 1-10. http://dx.doi.org/10.1016/0378-1135(90)90118-F

[9] Gonzalez, A., Arnaiz, I. and Yus, E. (2014) Evaluation of Long-Term Antibody Responses to Two Inactivated Bovine Viral Diarrhoea Virus (BVDV) Vaccines. Veterinary Journal, 199, 424-428. 
http://dx.doi.org/10.1016/j.tvj1.2013.12.005

[10] Lanyon, S.R., Anderson, M.L., Bergman, E. and Reichel, M.P. (2013) Validation and Evaluation of a Commercially Available ELISA for the Detection of Antibodies Specific to Bovine Viral Diarrhoea Virus (Bovine Pestivirus). Australian Veterinary Journal, 91, 52-56. http://dx.doi.org/10.1111/j.1751-0813.2012.01010.x

[11] Thobokwe, G., Heuer, C. and Hayes, D. (2004) Validation of a Bulk Milk Tank Milk Antibody ELISA to Detect Dairy Herds Likely Infected with Bovine Viral Diarrhoea Virus in New Zealand. New Zealand Veterinary Journal, 52, 394400. http://dx.doi.org/10.1080/00480169.2004.36457

[12] Humphry, R., Brulisauer, F., McKendrick, I., Nettleton, P.F. and Gunn, G.J. (2012) Prevalence of Antibodies to Bovine Viral Diarrhoea Virus in Bulk Tank Milk and Associated Risk Factors in Scottish Dairy Herds. Veterinary Record, 171, 445-452. http://dx.doi.org/10.1136/vr.100542

[13] Weir, A., Heuer, C., McDougall, S. and Voges, H. (2013) Use of an Enzyme-Linked Immunosorbent Assay for Detecting Bovine Viral Diarrhoea Virus Antibodies in Individual Cow Milk Samples. New Zealand Veterinary Journal, 61, 305-309. http://dx.doi.org/10.1080/00480169.2012.757729

[14] Jenvey, C., Reichel, M. and Cockcroft, P.D. (2014) Investigation of the Comparative Sensitivity of Serum, Colostrum and Whey for Detection of Specific Antibodies in Sheep Vaccinated against Johne's Disease. Small Ruminant Research, 123, 193-195. http://dx.doi.org/10.1016/j.smallrumres.2014.10.006

[15] Saah, A. and Hoover, D. (1997) "Sensitivity" and "Specificity" Reconsidered: The Meaning of These Terms in Analytical and Diagnostic Settings. Annals of Internal Medicine, 126, 91-94. http://dx.doi.org/10.7326/0003-4819-126-1-199701010-00026

[16] Lanyon, S., McCoy, R., Bergman, E. and Reichel, M. (2014) Milk as a Diagnostic Sample for a Commercially Available ELISA to Identify Bovine Viral Diarrhoea (BVD) Antibodies in Dairy Herds. Australian Veterinary Journal, 92, 269-273. http://dx.doi.org/10.1111/avj.12188

[17] Zervens, L., Nielsen, S. and Jungersen, G. (2013) Characterisation of an ELISA Detecting Immunoglobulin G to Mycobacterium avium subsp. Paratuberculosis in Bovine Colostrum. Veterinary Journal, 197, 889-891. http://dx.doi.org/10.1016/j.tvil.2013.03.018 
Scientific Research Publishing (SCIRP) is one of the largest Open Access journal publishers. It is currently publishing more than 200 open access, online, peer-reviewed journals covering a wide range of academic disciplines. SCIRP serves the worldwide academic communities and contributes to the progress and application of science with its publication.

Other selected journals from SCIRP are listed as below. Submit your manuscript to us via either submit@scirp.org or Online Submission Portal.
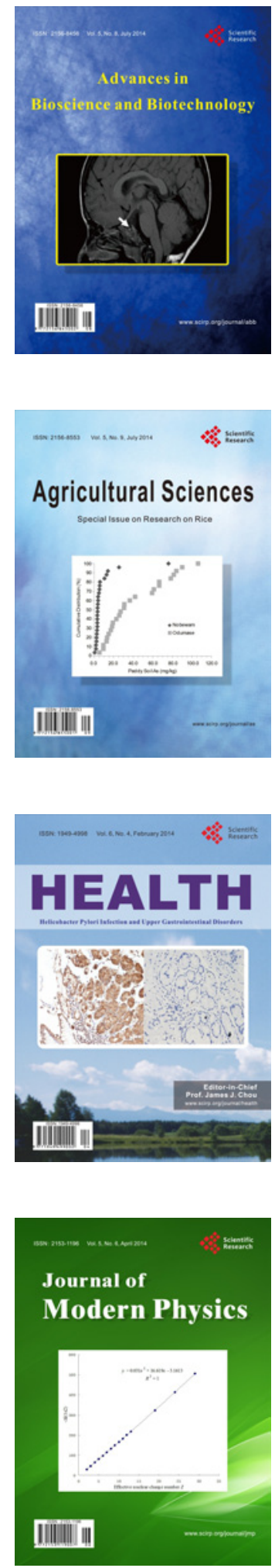
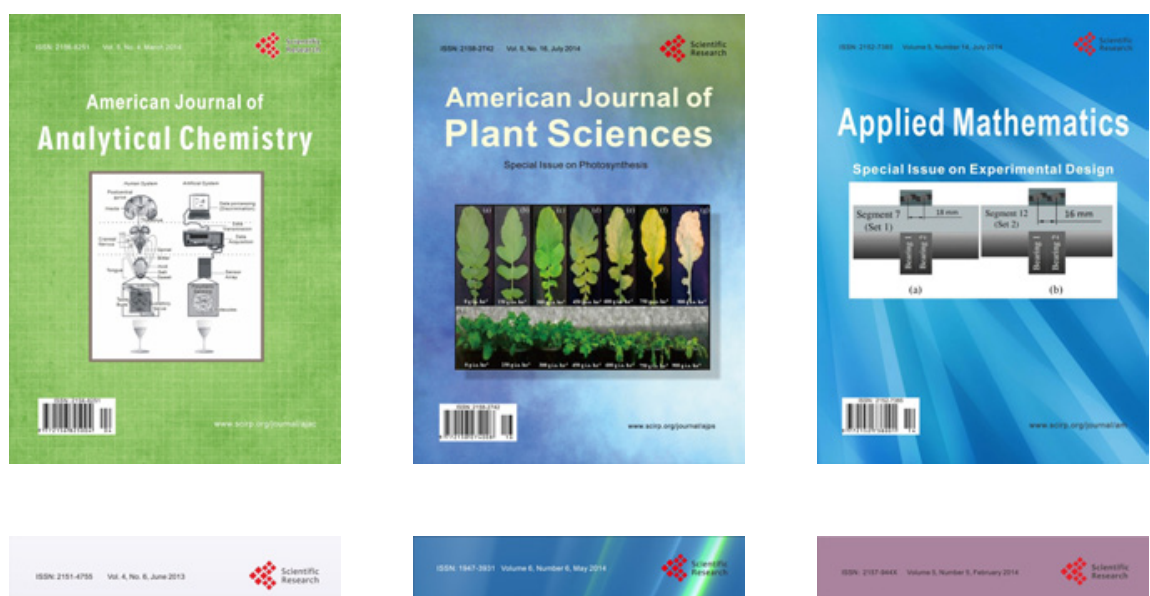

Creative Education
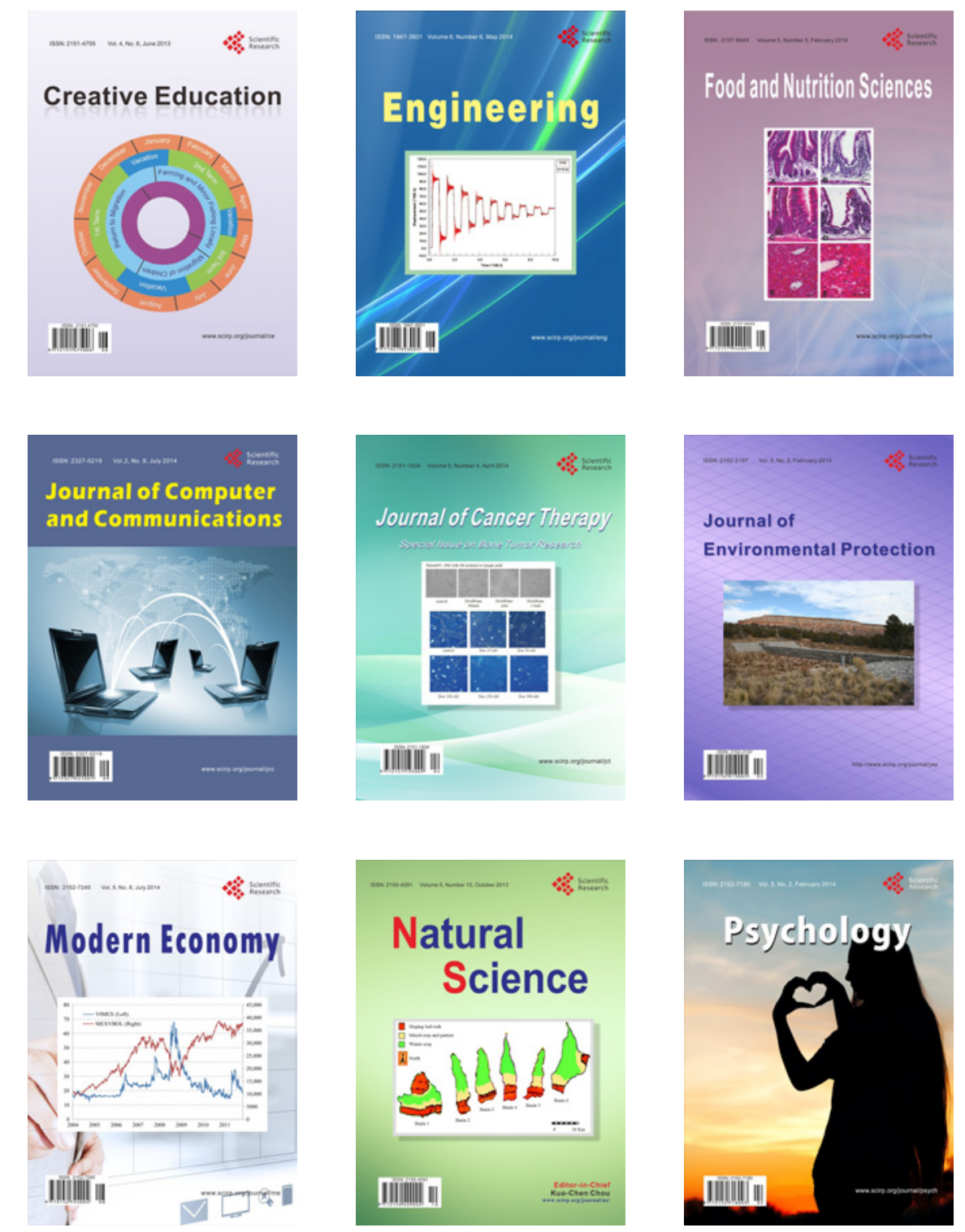